\title{
PRIMARY VAGINAL CANCER: A SINGLE INSTITUTION CASE SERIES OF PATIENT, TUMOUR AND TREATMENT FACTORS AFFECTING OUTCOMES
}

\author{
Obermair, H.M. ${ }^{1,}$; Harrison, M. ${ }^{1}$, Lim, K. ${ }^{1}$; Herbst, U. ${ }^{1}$ \\ 1 - Liverpool Hospital, South-West Sydney Local Health District, Sydney, NSW, Australia \\ 2 - University of New South Wales, Sydney, NSW, Australia
}

\begin{abstract}
Introduction
- Primary vaginal carcinomas are rare, accounting for $1-2 \%$ of gynaecological malignancies (1)

- Cases are classified as vaginal carcinomas only after the exclusion of cervical, urethral or vulval origins (2)

- Vaginal cancers generally occur in older women, with less than $15 \%$ of tumours occurring in patients under 40 years of age (3)

- More than $90 \%$ of vaginal cancer cases are squamous cell carcinomas

- Due to its rarity, data concerning the natural history, prognostic factors and treatment of vaginal carcinoma is mainly describes in small retrospective studies
\end{abstract}

\section{Aims \& Objectives}

- This project aimed to describe a series of primary vaginal cancer cases at a single institution, identifying patient, tumour and treatment factors impacting outcomes

\section{Methods}

- A retrospective chart analysis was performed for all patients diagnosed with primary vaginal cancer from 2008 to 2018 at a single institution in Australia

- For each case, data extracted included:

- Histopathology

- Staging

- Treatment received

- Socio-demographic factors

- Patient outcomes

\section{Results}

\section{Demographics}

- Average age $=59$ years (age range $34-75$ years)

- Average Charlson comorbidity index $=4.75$

- Average Body Mass Index (BMI) $=26.2$

- Index of Relative Socio-Economic Disadvantage $87.5 \%$ of patients fell into the most disadvantaged quintile

- Smoking status - 4 current smokers, 2 ex-smokers, 2 never-smokers

\section{Histopathology}

- $75 \%$ Squamous Cell Carcinomas

- $25 \%$ Melanoma

Stage at presentation

- Stage $1=3$ patients

- Stage $2=2$ patients

- Stage $3=3$ patients

- Stage $4=0$ patients

Management

- $62.5 \%$ of patients had surgery

- $50 \%$ had radiotherapy

- $50 \%$ had chemotherapy

- 1 patient also had immunotherapy

Outcomes

- At the end of follow-up period, $75 \%$ of patients alive, $25 \%$ deceased

- Average survival $=49.625$ months

- 5 year survival $=37.5 \%$

- $50 \%$ recurrence - all those who had recurrence had metastatic disease

\section{Conclusion}

This study indicates that outcomes for vaginal cancers are poor, and that early presentation is essential to improve outcomes. Treatment is highly individualised based on extent of disease at presentation. Due to limited literature in this area, it is difficult to compare patient demographics, tumour factors and standard treatment.

\section{References}

(1) Di Donato, V., Bellati, F., Fischetti, M., Plotti, F., Perniola, G., \& Panici, P. B. (2012). Vaginal cancer. Crit Rev Oncol Hematol, 81(3), $286-295$. doi:10.1016/j.critrevonc.2011.04.004

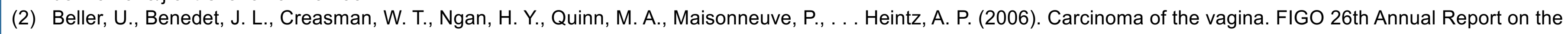
Results of Treatment in Gynecological Cancer. Int J Gynaecol Obstet, 95 Suppl 1, S29-42. doi:10.1016/s0020-7292(06)60029-5

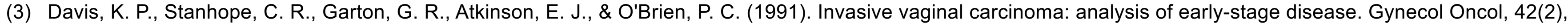
131-136. doi:10.1016/0090-8258(91)90332-y 